\title{
Editorial
}

\section{Computational Aspects of Social Network Analysis}

\author{
Przemyslaw Kazienko, ${ }^{1}$ Reda Alhajj, ${ }^{2}$ and Jaideep Srivastava ${ }^{3}$ \\ ${ }^{1}$ Wroclaw University of Technology, 50-370 Wroclaw, Poland \\ ${ }^{2}$ University of Calgary, Calgary, AB, Canada T2N 1N4 \\ ${ }^{3}$ University of Minnesota, Minneapolis, MN 5545, USA \\ Correspondence should be addressed to Przemyslaw Kazienko; przemyslaw.kazienko@pwr.edu.pl
}

Received 12 March 2015; Accepted 12 March 2015

Copyright (C) 2015 Przemyslaw Kazienko et al. This is an open access article distributed under the Creative Commons Attribution License, which permits unrestricted use, distribution, and reproduction in any medium, provided the original work is properly cited.

The interdisciplinary research field commonly called Social Network Analysis (SNA) has attracted many scientists from various disciplines from physics, sociology, anthropology, psychology, management, and also computer science. Various methodologies used in different disciplines are complementary to computational methods used to process data about human activities, profiles, or direct social relationships.

Users of IT systems leave their traces in most of these systems and the Internet is the largest source of data about human behavior, mutual interactions, and collaboration. It refers especially to social media like Twitter or Facebook as well as many other Web 2.0 services. This data is widely utilized to study social phenomenon and if they also respect social relationships, then we can say that SNA method is applied there.

It includes seven papers that come closer to various problems related to efficient processing of large social networks, link prediction, community detection, and analysis of human behavior and skills.

The paper entitled "A Community Based Approach for Link Prediction in Signed Social Networks" provides methods to predict the sign of human relationships: either positive or negative. These approaches are based on analysis of stable social communities and creation of appropriate node reputation rankings. The experiments on real data sets proved the high accuracy of the approach.

The paper entitled "Parallelizing SLPA for Scalable Overlapping Community Detection" is also related to social communities, in particular detection of overlapping groups in the large-scale environment. The extraction of overlapping communities is typically more computationally intensive than disjoint ones, so it requires more sophisticated solutions. The authors proposed multithreaded SLPA algorithm that is almost linearly scalable and provides high quality overlapping social communities, which was confirmed on large real social networks.

The paper entitled "Fast Parallel All Subgraph Enumeration Using Multicore Machines" describes computational methods for efficient enumeration of all subgraphs for a given social network graph. Similar to the previous paper, it operates in the parallel, multicore environment in order to enable processing of large social networks. The method proposed makes use of polynomial heuristic for subgraph isomorphism detection to prune candidate subgraphs and reduce necessary operations.

The paper presents Skillrank, a hybrid method to assess quality and confidence of professional skills in social networks. Experts and their expertise are detected, verified, and ranked using specialized trust metrics. It makes use of various pieces of information available within the professional social network: human relationships and endorsements as well as user profiles. The authors have shown that network-based methods can be very effective in accurate prediction and valuation of human skills.

The paper entitled "Link Prediction Methods and Their Accuracy for Different Social Networks and Network Metrics" investigates the correlation between network metrics and accuracy of various link prediction methods. The authors analysed ten different methods for prediction of existence of a new link in the social network and tried to observe which 
of them are more suitable for different network types, that is, real social networks with a certain structural profile.

The paper "On Efficient Link Recommendation in Social Networks Using Actor-Fact Matrices" deals with the same problem as the previous paper, prediction of new links in the social network. The authors claim that the computation quality of link recommendation algorithms significantly depends on the social network representation. They found out, in particular, that the actor-fact matrix appears to be the best model for the link recommendation problem.

Finally, computational comparison of human activities online was performed in the last manuscript entitled "The Comparison of Users Activity on the Example of Polish and American Blogosphere." The authors compared two separate online communities: users of Polish and American blogospheres and found significant quantitative differences, also in the dynamics of human habits.

The readers will enjoy reading this special issue to get exposed to various aspects and applications of SNA as presented by some leading researchers in the field.

\section{Acknowledgments}

The work was partially supported by The National Science Centre, decision no. DEC-2013/09/B/ST6/02317, and the European Commission under the 7th Framework Programme, Coordination and Support Action, Grant Agreement no. 316097, the ENGINE project.

Przemyslaw Kazienko Reda Alhajj

Jaideep Srivastava 

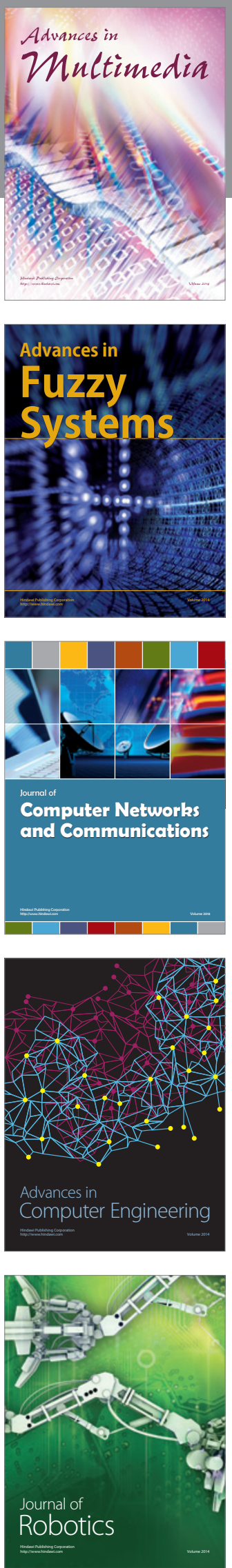

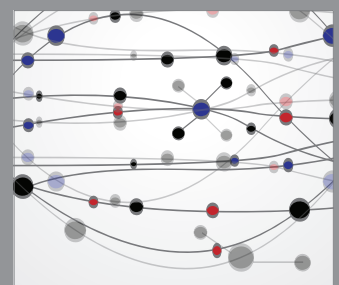

The Scientific World Journal
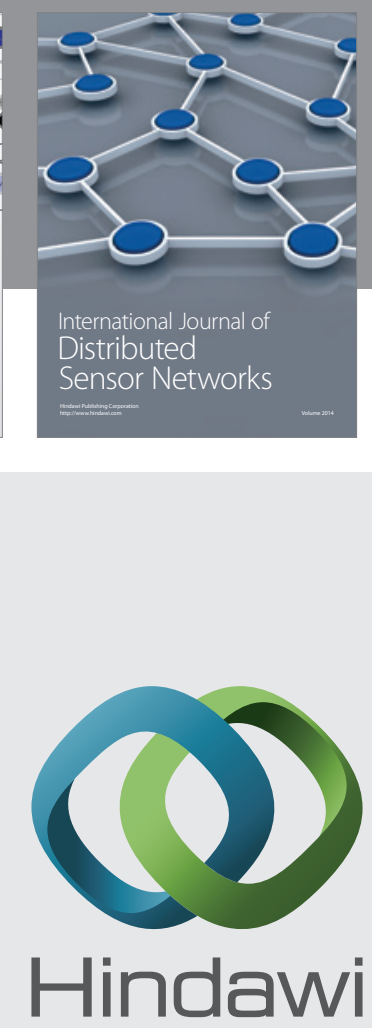

Submit your manuscripts at

http://www.hindawi.com
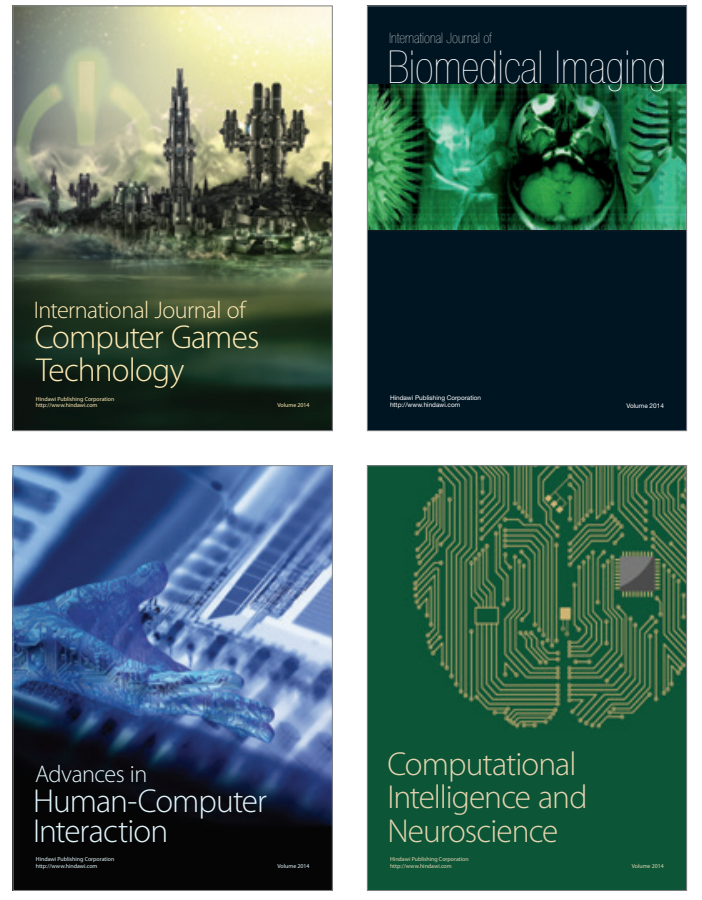
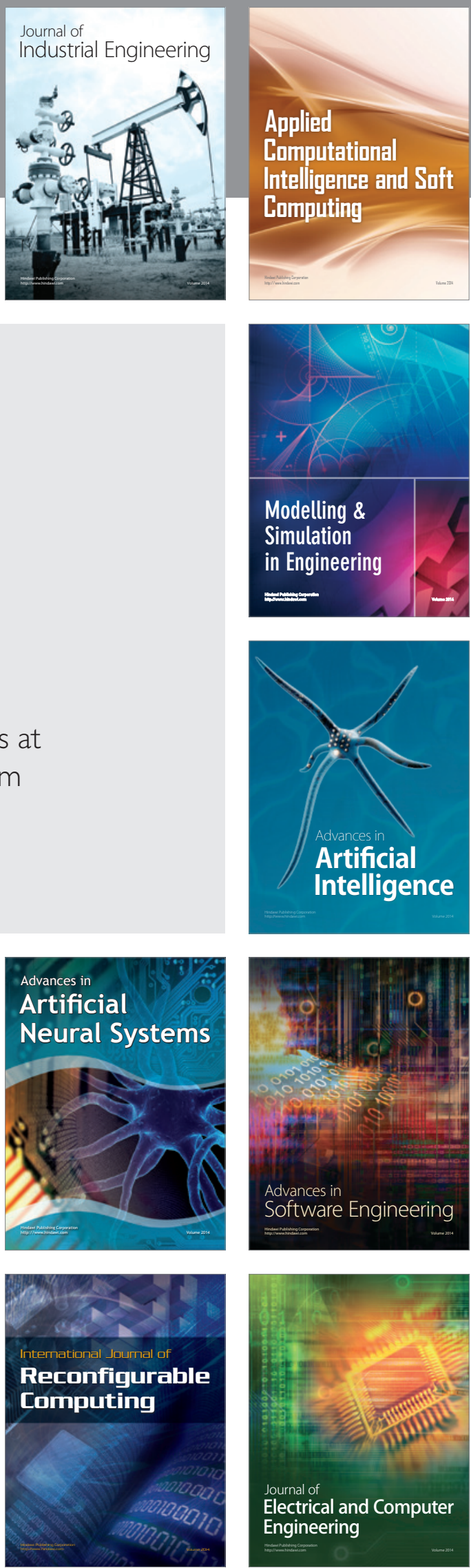